\title{
Prevalência de alterações radiológicas nas articulações do tarso em equinos com sobrepeso da raça crioula em manejo extensivo
}

\author{
Prevalence of juvenile osteoarthritis tarsal joints in overweight crioulo breed mares in extensive \\ nutritional management
}

Prevalencia de alteraciones radiológicas en las articulaciones tarsales em caballos criollos con sobrepeso criados en manejo extensivo

\section{Resumo}

A busca intensiva por um manejo nutricional eficaz fez com que animais de diferentes categorias apresentassem sinais de sobrepeso. A obesidade é citada em diferentes raças como a causa de inúmeros problemas ortopédicos como laminite, osteocondrite dissecante e osteoartrite juvenil. O objetivo deste trabalho é avaliar por meio da avaliação radiográfica, a presença de alterações nas articulações do tarso de animais nascidos e criados em manejo extensivo em um criatório da raça Crioula, e que se mantinham em sobrepeso durante o ano todo. Foi realizado um estudo retrospectivo de coorte em condições de campo de fêmeas com sobrepeso e/ou obesidade desde o nascimento, até a idade adulta. Foram avaliadas 37 fêmeas equinas da raça Crioula, nascidas e criadas nesta mesma propriedade. Foi realizada a pesagem com fita e mensuração da gordura da base da cauda com a utilização de aparelho de ultrassonografia. As projeções radiográficas foram confeccionadas na região do tarso em ambos membros pélvicos. As éguas apresentaram média de escore de condição corporal 8, média de peso 557,29 +13,66 kg, gordura da base da cauda de 30,54 +3,17 mm. Cerca de 62,16\% dos equinos apresentaram achados radiológicos, com alterações de diminuição de espaço articular, proliferações ósseas e osteófitos classificadas em grau 2, 3 e 4. Nos animais de um ano de idade (12 meses), foi observado $70 \%$ de alterações articulares nos ossos do tarso. Tanto a obesidade nas éguas em reprodução, quanto o sobrepeso nos animais em desenvolvimento, podem ser fatores etiológicos importantes para o desenvolvimento desta doença na raça Crioula.

Palavras-chave: Osteoartrite juvenil; Sobrepeso; Tarso; Raça crioula.

\begin{abstract}
Breeders look for effective nutritional management, but, behind it, this management causes horses with signs of overweight between different ages. Obesity is mentioned in several horses breeding as the cause of numerous orthopedic problems such as laminitis, osteochondritis dissecans and juvenile osteoarthritis. The aims of this research is to evaluate, through radiographic examination, signs of possible osteoarticular disease in tarsal joints of overweight
\end{abstract}


Crioulo females, which was foaled and bred at a particular farm in extensive management. A retrospective cohort study was performed out at field conditions with overweight and/or obese equine females, from birth until adulthood. It was evaluated thirty-seven Crioulo females, which were foaled and bred at the same particular farm. All animals were weighed measured with a scale tape and the fat tailhead was measured through ultrasound examination. The radiographic projections of the tarsal joints region were realized in both pelvic limbs. Crioulo females presented average body condition score (BCS) of 8; average body weight of 557,29 kg; average fat tailhead of 30,54 +3,17 mm. About of $62,16 \%$ of the total females were radiological characterized in having decreased of the articular space, bone proliferation and articular osteophytes of the tarsal joints. Those articular changes were classified as grade 1, 2, 3 or 4, according to the severity. Articular changes in the tarsal joint were observed in $70 \%$ of 12 ' months aged fillies. Both, mares' obesity and growing fillies' overweight, can be an important ethiological factor to the development of tarsal articular diseases in the Crioulo breed.

Keywords: Osteochondritis dissecans; Overweight; Tarsal articular; Crioulo breed.

\section{Resumen}

La búsqueda intensiva de un manejo nutricional efectivo provocó que animales de diferentes categorías mostraran signos de sobrepeso. La obesidad se cita en diferentes razas como la causa de numerosos problemas ortopédicos como laminitis, osteocondritis disecante y osteoartritis juvenil. El objetivo de este estudio es analizar, mediante evaluación radiográfica, la presencia de alteraciones en las articulaciones tarsales de animales nacidos y criados en manejo extensivo en la raza Crioula, y que permanecieron con sobrepeso durante todo el año. Se realizó un estudio de cohorte retrospectivo en condiciones de campo de yeguas con sobrepeso y / u obesidad desde el nacimiento hasta la edad adulta. Se evaluaron 37 hembras equinas Crioula, nacidas y criadas en esta misma propiedad. Se pesó con una cinta y se midió la grasa en la base de la cola utilizando un dispositivo de ultrasonido. Se realizaron proyecciones radiográficas en la región tarsiana de ambas extremidades pélvicas. Las yeguas tenían una puntuación media de condición corporal de 8, peso medio 557,29 + 13,66 kg, grasa de la base de la cola de 30,54 $+3,17 \mathrm{~mm}$. Aproximadamente el 62,16\% de los caballos presentaba hallazgos radiológicos, con alteraciones de espacio articular reducido, proliferación ósea y osteofitos clasificados como grado 2, 3 y 4. En animales de un año (12 meses), $70 \%$ de alteraciones articulares en el tarso huesos. Tanto la obesidad en las yeguas reproductoras como el sobrepeso en los animales en desarrollo pueden ser factores etiológicos importantes para el desarrollo de esta enfermedad en la raza Crioula.

Palabras clave: Artrosis juvenil; Sobrepeso; Tarso; Raza criolla.

\section{Introdução}

No início do século 20, a raça Crioula foi selecionada por critérios zootécnicos, agregando valor aos animais. A partir do final dos anos 80, a indústria do cavalo Crioulo se desenvolveu com o surgimento de provas funcionais e exposições morfológicas, havendo a necessidade de animais mais precoces e resultados imediatos (Pimentel, 2017).

A busca intensiva por um manejo nutricional eficaz fez com que animais de diferentes categorias apresentassem sinais de sobrepeso e obesidade. A obesidade é citada em diferentes raças como a causa de inúmeros problemas ortopédicos como laminite, osteocondrite dissecante e osteoartrite juvenil (Amaral et al., 2017; Gallio et al., 2014; Heyden, et al., 2012; Peugnet, et al., 2016). Segundo Abreu et al. (2011), 76,47\% das claudicações de membros pélvicos em cavalos crioulos em treinamento tinham origem nas articulações de tarso. A osteoartrite juvenil das articulações de tarso é considerada uma doença ortopédica de desenvolvimento em animais jovens (McIlwraith, 2004). A principal causa desta alteração em potros é a alimentação rica em carboidrato, que altera o metabolismo da insulina, responsável pela maturação dos condrócitos (Pagan et al., 2001; Ralston, 1996; Savage e Jeffcott, 1993).

Em um estudo com animais crioulos de até 26 meses e estabulados, demonstrou que 79,2\% dos equinos apresentavam osteoartrose em articulações do tarso (Gallio et al., 2014), enquanto que, em outro estudo com animais de 18 meses de idade, 100\% dos animais apresentaram esta mesma alteração (Amaral, et al., 2017). Segundo Torres et al. (2020), baixos níveis séricos do hormônio de crescimento (IGF 1) durante o período de desenvolvimento de potros da raça Crioula esteve associado a degenerações cartilaginosas das articulações társicas.

O objetivo deste trabalho é avaliar por meio da avaliação radiográfica, a presença de alterações nas articulações do tarso de animais nascidos e criados em manejo extensivo em um criatório da raça Crioula, e que se mantinham em sobrepeso durante o ano todo. A hipótese é de que animais criados e mantidos em sobrepeso oriundo de éguas com sobrepeso 
desenvolvam osteoartrite das articulações do tarso, independente de outros fatores como por exemplo, genéticos e sobrecarga articular realizada pelo exercício.

\section{Metodologia}

Este estudo foi realizado em uma propriedade no município do Capão do Leão/RS, $24 \mathrm{~km}$ a oeste da cidade de Pelotas/RS, latitude $31^{\circ} 72^{\prime \prime} 25^{\prime} \mathrm{S}$, longitude $52^{\circ} 55^{\prime \prime} 83^{\prime} \mathrm{O}$. Foi realizado um estudo retrospectivo de coorte em uma pesquisa de campo de fêmeas com sobrepeso e/ou obesidade desde o nascimento, até a idade adulta.

Foram avaliadas 37 fêmeas equinas da raça Crioula entre um e onze anos de idade, nascidas e criadas nesta mesma propriedade. No manejo da propriedade, as éguas são mantidas a campo com pastagem natural melhorada com azevém (Lolium multiflorum). Este tipo de forragem é cultivada no mês de março, equivalente ao final do verão nesta região, possui crescimento rápido para pastoreio, e se mantém viável até o mês de dezembro, equivalente a entrada do verão. Ou seja, os animais permanecem a campo com esta forrageira durante nove meses do ano. $\mathrm{O}$ único momento em que esses animais a campo não são alimentados com este tipo de forrageira é durante os meses do verão, de janeiro a março, onde prevalece o campo nativo. A pastagem de Lolium multiflorum apresenta maior desenvolvimento durante a primavera (setembro e outubro) concentrando 72,4 e $67,1 \%$ da sua produção total de matéria seca neste período contra 27,6 e 32,9\% no período de outonoinverno correspondendo aos meses de maio, junho, julho e agosto (Alves-Filho et al., 2003).

Todos os animais desta propriedade são submetidos a doma com dois anos de idade e participam de exposições morfológica da raça Crioula a partir desta idade. Contudo, nenhum dos animais da propriedade é preparado para participar de provas de avaliação funcional de forma a ser submetido a treinamento atlético. A partir dos quatro anos de idade, todos os animais são mantidos em sistema extensivo a campo e são destinados a reprodução.

As éguas foram divididas em categorias de acordo com a idade, sendo estas: um ano de idade $(n=10)$, dois a três anos de idade $(n=8)$ e quatro a onze anos de idade $(n=19)$. Foi realizada a pesagem de todos os animais por meio, da utilização de fita balança e o escore de condição corporal (ECC) dos animais de acordo, com a escala de 1-9 proposta por Henneke, Potter e Krieder (1983), na qual 1 significa extremamente magro e 9 extremamente obeso. Além disso, foi realizada a mensuração da gordura da base da cauda de acordo com o protocolo descrito por Gentry et al. (2004) com a utilização de aparelho de ultrassonografia Mindray 2200 acoplado a uma probe linear.

As projeções radiográficas dorso-plantar, latero-medial, dorso lateral plantaro medial obliqua $45^{\circ}$ e dorso medialplantaro lateral obliqua $45^{\circ}$ foram confeccionadas na região do tarso em ambos membros pélvicos. $\mathrm{O}$ aparelho de radiografia digital utilizado foi um DR EcoRay. Alterações como artropatias das articulações do tarso evidenciadas em ao menos um dos membros pélvicos foi considerada para classificação das lesões articulares de acordo com a escala proposta por Robert, Valette e Denoix (2006), onde o grau 1 é definido como sem ou pouco significado radiológico, grau 2 com leves achados anormais radiológicos, grau 3 representa moderados achados anormais radiológicos e grau 4 descreve graves achados anormais radiológicos (Tabela 1).

Tabela 1. Classificação das lesões radiográficas.

\begin{tabular}{cc}
\hline Grau & Descrição \\
1 & Sem ou pouco significado radiológico \\
2 & Leves achados anormais radiológicos \\
3 & Moderados achados anormais radiológicos \\
4 & Graves achados anormais radiológicos \\
\hline
\end{tabular}

Fonte: Robert et al. (2006). 
O ECC (escore de condição corporal), o peso corporal $(\mathrm{kg})$ e a medida de gordura da base da cauda $(\mathrm{mm})$ para cada categoria de idade estão descritas na Tabela 2. A análise estatística foi realizada com o software comercial IBM SPSS statistics 23, utilizando análise descritiva dos dados. Os dados estão expressos em média + desvio padrão.

Tabela 2. Médias \pm desvio padrão de peso $(\mathrm{kg})$, gordura da base da cauda $(\mathrm{mm})$ e escore de condição corporal (ECC), distribuídas por idade.

\begin{tabular}{cccc}
\hline Categoria & Média do Peso & Média Gord Base Cauda & Média ECC \\
Animais de 1 ano $(\mathrm{n}=10)$ & $317,62( \pm 30,46) \mathrm{kg}$ & $16,62( \pm 1,16) \mathrm{mm}$ & 7 \\
Animais de 2 a 3 anos $(\mathrm{n}=8)$ & $389,66( \pm 30,17) \mathrm{kg}$ & $16,5( \pm 2,39) \mathrm{mm}$ & 7 \\
Animais de 4 a 11 anos $(\mathrm{n}=19)$ & $557,29( \pm 27,53) \mathrm{kg}$ & $30,54( \pm 3,98) \mathrm{mm}$ & 8 \\
\hline
\end{tabular}

Fonte: Autores (2021).

\section{Resultados e Discussão}

As classificações das lesões articulares encontradas em cada categoria estão descritas na Tabela 3 de acordo com o grau de lesão, segundo a classificação de Robert, Valette e Denoix (2006). Observou-se na categoria referida a 1 ano de idade, uma maior porcentagem (50\%) nas lesões de grau 2, diferente das categorias de 2 a 3 anos e 4 a 11 anos, onde a maior porcentagem foi observada em leões de grau 1 (Tabela 3).

Tabela 3. Distribuição das alterações radiográficas por idade.

\begin{tabular}{lccccccccc}
\hline & \multicolumn{2}{c}{ Grau 1 } & \multicolumn{2}{c}{ Grau 2 } & \multicolumn{2}{c}{ Grau 3 } & Grau 4 & \multicolumn{2}{c}{ Total (n) } \\
\multicolumn{1}{c}{ Categoria } & $\mathrm{N}$ & $\%$ & $\mathrm{n}$ & $\%$ & $\mathrm{n}$ & $\%$ & $\mathrm{n}$ & $\%$ & \\
1 ano & $3 / 10$ & $30 \%$ & $5 / 10$ & $50 \%$ & $1 / 10$ & $10 \%$ & $1 / 10$ & $10 \%$ & 10 \\
2 a 3 anos & $3 / 8$ & $37,5 \%$ & $1 / 8$ & $12,5 \%$ & $2 / 8$ & $25 \%$ & $2 / 8$ & $25 \%$ & 8 \\
4 a 11 anos & $8 / 19$ & $42,10 \%$ & $2 / 19$ & $10,52 \%$ & $2 / 19$ & $10,52 \%$ & $7 / 19$ & $36,84 \%$ & 19 \\
\hline Total & $18 / 37$ & $37,83 \%$ & $8 / 37$ & $21,62 \%$ & $5 / 37$ & $13,51 \%$ & $10 / 37$ & $27,02 \%$ & 37 \\
\hline
\end{tabular}

Fonte: Autores (2021).

A presença de alterações leves, moderadas e severas estão descritas na Tabela 4, destacando-se uma maior porcentagem de alterações moderadas e severas $(62,16 \%)$ em comparação as alterações leves $(37,83 \%)$ no total das categorias.

Tabela 4. Distribuição das alterações radiográficas anormais por idade.

\begin{tabular}{lccccc}
\hline & \multicolumn{2}{c}{$\begin{array}{c}\text { Alteração leve } \\
\text { (grau 1) }\end{array}$} & $\%$ & $\mathrm{~N}$ & Alterações moderadas e severas \\
Categoria & $\mathrm{N}$ & $30 \%$ & 7 & $\%$ & Total (n) \\
1 ano & 3 & $37,5 \%$ & 5 & $70 \%$ & 10 \\
2 a 3 anos & 3 & $42,10 \%$ & 11 & $62,5 \%$ & 8 \\
4 a 11 anos & 8 & $37,83 \%$ & 23 & $57,89 \%$ & 19 \\
\hline Total & 14 & & $62,16 \%$ & 37 \\
\hline
\end{tabular}

Fonte: Autores (2021). 
A Figura 1 representa a alteração radiológica de classificação grau 1, onde observa-se a presença de um pequeno osteófito localizado na articulação tarso metatarsiana.

Figura 1. Projeção radiográfica dorso medial-plantaro lateral obliqua $45^{\circ}$ : classificação radiográfica de grau 1 . Observa-se pequeno osteófito (seta) da articulação tarso metatarsiana.

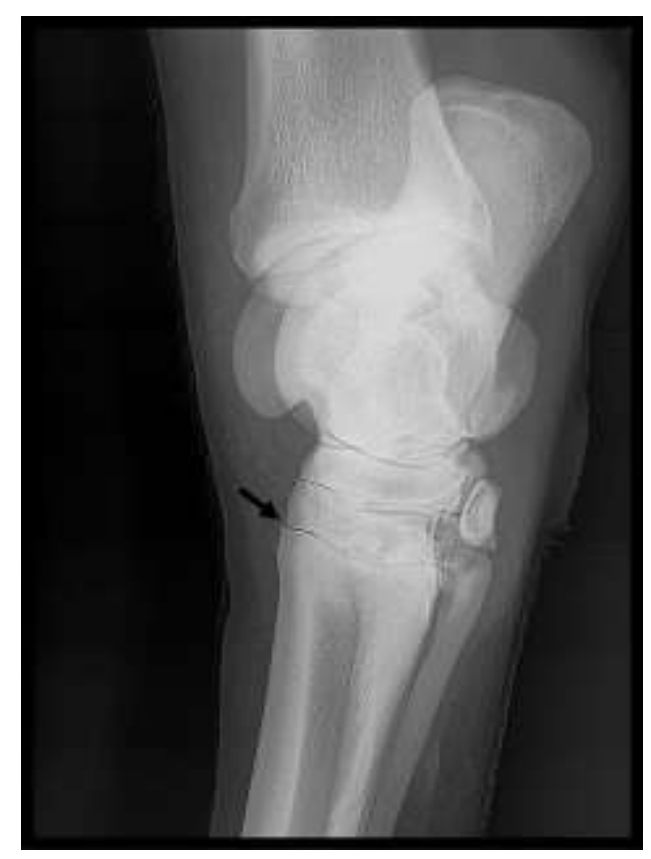

Fonte: Autores (2021).

As alterações radiográficas de classificação 2 são representadas pela Figura 2, onde observa-se na articulação tarso metatarsiana leve diminuição do espaço articular associado a formação de osteófito. Somado a presença de proliferação óssea e diminuição do espaço articular da intertársica distal. 
Figura 2. Projeção radiográfica dorso medial-plantaro lateral obliqua $45^{\circ}$ : classificação radiográfica de grau 2. Observa-se leve diminuição de espaço articular associado a formação de osteófito (seta preta) da articulação tarso metatarsiana. Proliferação óssea associada a diminuição de espaço articular da articulação intertársica distal (seta vermelha).

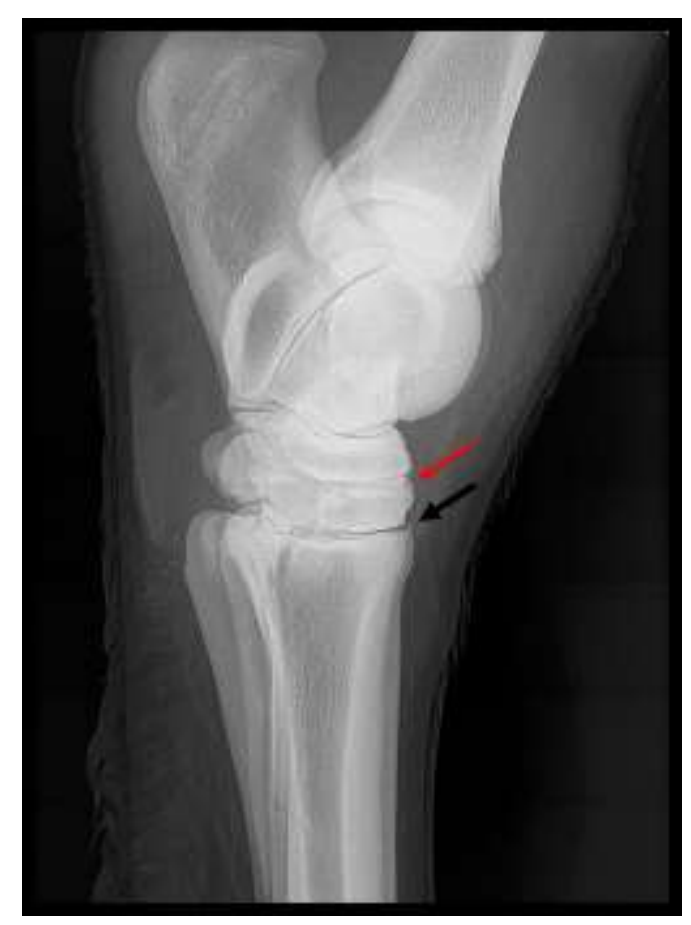

Fonte: Autores (2021).

Na classificação radiográfica de grau 3 é possível observar uma proliferação óssea associado a diminuição severa do espaço articular da articulação intertársica distal (Figura 3).

Figura 3. Projeção radiográfica dorso medial-plantaro lateral obliqua $45^{\circ}$ : classificação radiográfica de grau 3. Observa-se proliferação óssea com diminuição severa de espaço articular (seta) da articulação intertársica distal.

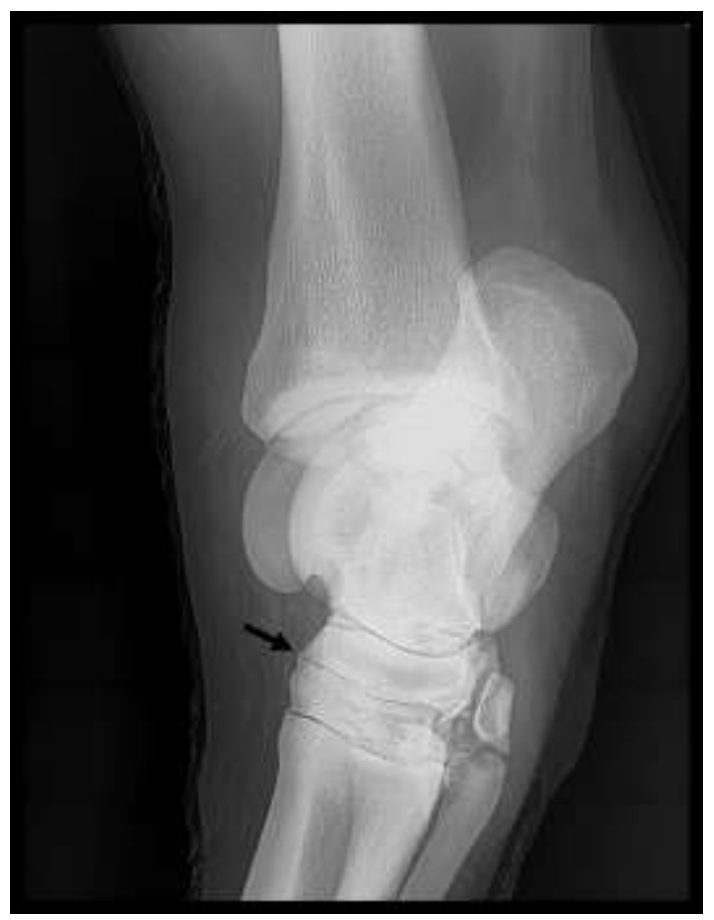

Fonte: Autores (2021). 
Na Figura 4 observa-se a presença de esparavão ósseo na articulação tarso metatarsiana, associado a diminuição severa do espaço articular da articulação intertársica distal e presença de um fragmento na tróclea do talus medial, caracterizando uma alteração radiográfica de grau 4.

Figura 4. Projeção radiográfica dorso medial-plantaro lateral obliqua $45^{\circ}$ : classificação radiográfica de grau 4 . Observa-se esparavão ósseo (seta preta) da articulação tarso metatarsiana caracterizada por um pequeno fragmento ósseo não deslocado. A articulação intertársica distal (seta vermelha) com diminuição severa de espaço articular. Fragmento na tróclea do talus medial não deslocado (círculo azul).

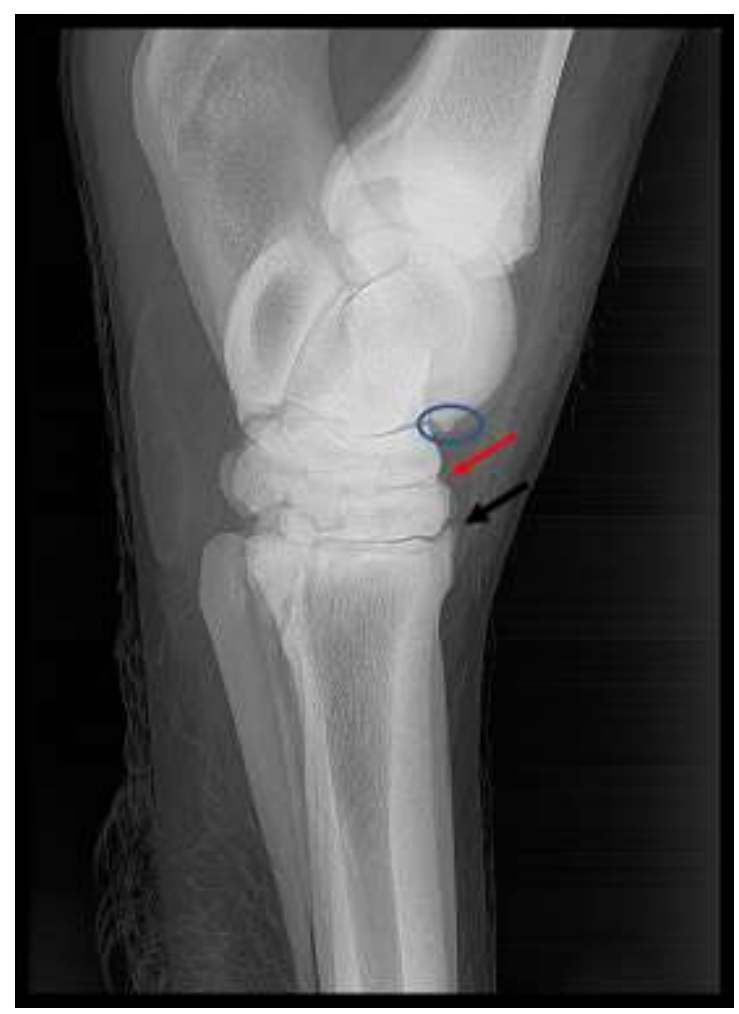

Fonte: Autores (2021).

A prevalência de artropatias da articulação intertársica proximal, intertársica distal e tarso metatarsiana demonstraram neste criatório com manejo extensivo 62,16\% de achados radiológicos, com alterações de diminuição de espaço articular, proliferações ósseas e osteófitos classificadas em grau 2, 3 e 4 em animais que nunca passaram por treinamento atlético. Sugerindo desta forma que, o manejo nutricional proposto a esses animais resultou em sobrepeso e/ou obesidade e que os mesmos podem estar envolvidos no desenvolvimento da doença. Resultados similares aos de Heyden et al. (2012), que também observaram significante aumento no risco de doença articular em potros aos 21 meses da raça Warmblood que foram suplementados com concentrado até um ano de idade e também em potros gerados de éguas que foram suplementadas com concentrado durante a gestação. Porém, no presente estudo, as éguas adultas gestantes (entre quatro e onze anos de idade) não foram suplementadas com concentrado, diferente do estudo de Heyden et al. (2012), onde foram mantidas em sistema extensivo a campo. Contudo, estas éguas, mesmo em sistema a campo e sem suplementação apresentam média de escore de condição corporal 8, média de peso 557,29 +13,66 kg, gordura da base da cauda de 30,54 +3,17 mm e ECC > 8, compatíveis com a obesidade para éguas da raça Crioula, segundo Paz et al. (2013) e Marchiori et al. (2015). Gomes e Reis (1997), observaram uma concentração de produção de forragem relativa para o azevém de 56\% no período da primavera, $30 \%$ no inverno e $14 \%$ no outono. Esta diferença na distribuição estacional é verificada em função de particularidades às condições 
climáticas, conforme ano agrícola e épocas de semeadura.

Peugnet et al. (2016), sugerem que alterações metabólicas nas éguas no terço final de gestação podem causar alterações epigenômicas nos potros. Segundo Torres et al. (2020), alterações radiográficas com prevalência de 92,5\% em lesões de tarso e 50\% de displasia fiseal em epífese distal de metacarpo em potros com 6 meses de idade estiveram associadas a diminuição nos níveis de 290 a $89 \mathrm{ng} / \mathrm{mL}$ de IGF 1 e a obesidade de suas mães no terço final da gestação. Robles et al. (2018) observaram que éguas Anglo-árabes e de Sela Francesa obesas durante a gestação aumentam o risco de produzirem potros com lesões articulares observadas aos 12 meses de vida destes, o que corrobora com os achados do presente estudo, em que as éguas em reprodução são observadas em escore corporal compatível com obesidade e seus potros, aos 12 meses, apresentam alta prevalência de lesões articulares (70\%).

A alta prevalência de alterações radiográficas de tarso observada em nosso estudo na categoria de animais de dois a três anos de idade (62,50\% com achados radiológicos anormais), corrobora com os achados de Gallio et al. (2014) e Amaral et al. (2017), em animais da raça Crioula de até 26 meses de idade, que encontraram a mesma doença nas mesmas articulações em 79,2\% e 100\%, respectivamente, de animais estabulados e alimentados com carboidrato. Os mesmos autores associaram as dietas ricas em energia e carboidratos e a obesidade como fatores predisponentes de osteoartrose em animais jovens. No presente estudo, observamos escore de condição corporal 7 nesta categoria de animais, identificando sobrepeso, segundo Paz et al. (2013) e Marchiori et al. (2015), mas não obesidade. Nos animais de manejo intensivo de até 18 meses de idade com alimentação rica em carboidratos que Amaral et al. (2017) avaliaram, a média da gordura subcutânea da cauda foi de 10,5 mm, enquanto que, no presente estudo, na categoria de 2 a 3 anos de idade, a gordura do mesmo local teve medida de 16,5+2,39 mm. Estes dados podem ser explicados pelo fato dos animais avaliados por Amaral et al. (2017) serem mantidos estabulados e sob um certo regime de exercícios para preparo de exposições morfológicas, enquanto que os animais do presente estudo eram mantidos a campo no momento do estudo e sem nenhum tipo de exercício forçado, o que levaria a um acúmulo maior de gordura subcutânea.

Nos animais de um ano de idade (12 meses) do presente estudo, foi observado $70 \%$ de alterações articulares nos ossos do tarso. Em um estudo com potros Lusitanos, observou-se lesões articulares em 76,08\% dos potros da mesma idade, porém, apenas 16,2\% desses potros mantiveram as lesões quando atingiram 18 meses de vida (Baccarin et al., 2011). Os potros Lusitanos referidos não foram identificados quanto ao escore de condição corporal e, portanto, não devem ser considerados em sobrepeso como em nosso estudo. Os animais de 1 ano de idade do presente estudo não foram reexaminados posteriormente para diagnosticar a permanência ou resolução das lesões encontradas, porém, nos demais animais deste estudo, com 2 a 3 anos de idade, a prevalência da doença segue em índices parecidos (62,5\%), o que sugere que as lesões tendem a permanecer nesses animais nos anos subsequentes de desenvolvimento.

\section{Conclusão}

62,16\% de animais da raça Crioula em sobrepeso e/ou obesidade neste manejo nutricional extensivo a campo possuem osteoartrite de tarso, mesmo sem terem sidos submetidos a treinamento atlético. Tanto a obesidade nas éguas em reprodução, quanto o sobrepeso nos animais em desenvolvimento, podem ser fatores etiológicos importantes para o desenvolvimento desta doença na raça Crioula.

\section{Ethical approval}

This work involved the use of non-experimental animals only (owned or unowned). Ethical approval from a committee was not necessarily required. 


\section{Declaration of interest}

The authors report no conflicts of interest.

\section{Referências}

Abreu H. C., De La Corte F. D., Brass K. E., Pompermayer E., da Luz T. R. R. \& Gasperi D. 2011. Claudicação em cavalos Crioulos atletas. Ciência Rural. 41(12): p. 2114-2119.

Alves-Filho D., Neumann M., Restle J., De Souza A. N. M. \& Peixoto L. A. O. 2003. Production, quality and the production cost of forage of ryegrass (Lolium multiflorum Lam) fertilized with two types of fertilizer. Ciência Rural. 33(1): 143-149.

Amaral L. A., Marchiori M., Moraes B. S., Finger I., dos Santos R. S. \& Nogueira, C. E. W. N. 2017. Relação entre adiposidade, perfil energético, proteínas inflamatórias e lesões osteoarticulares em equinos jovens sobre diferentes sistemas de criação. Pesquisa Veterinária Brasileira. 37(2): 115-120.

Baccarin R. A. Y., Pereira M. A., Roncati N. V., Furtado P. V., Oliveira C. A. \& Hagen S. C. F. 2011. Identificação dos níveis séricos do fator de crescimento tipo insulina 1 em potros com osteocondrose. Pesquisa Veterinária Brasileira. 31(8): 677-682.

Gallio M. I., Azevedo M. S., Brass K. E., De La Corte F. D. \& Lopes L. F. D. 2014. Prevalência de alterações ósseas no tarso de potros Crioulos de até vinte e seis meses de idade. Ciência Rural. 44 (8).

Gentry L. R., Donald L., Thompson J. R., Glen T., Gentry J. R., Ronald P., Del Vecchio K. A. \& Del Vecchio P. M. 2004. The Relationship Between Body Condition Score and Ultrasonic Fat Measurements in Mares of High Versus Low Body Condition. Journal Equine Veterinary Science. (24):198-203.

Gomes J. F. \& Reis J. C. L. 1997. Produção de forrageiras anuais de estação fria no Litoral sul do Rio Grande do Sul. In: Reunião Anual da Sociedade Brasileira de Zootecnia (Juiz de Fora, Brasil). pp.83-85.

Heyden V. L., Lejeune J-P., Caudron I., Detilleux J., Sandersen C., Chavatte P., Paris J., Deliège B. \& Serteyn D. 2012. Association of breeding conditions with prevalence of osteochondrosis in foals. Veterinary Record. (172):68.

Henneke D. R., Potter G. D. \& Krieder J. L. 1983. Relationship between condition score, physical measurements and body fat percentage in mares. Equine Veterinary Journal. 15: 371-372.

Marchiori M. O., Kasinger S., Silva K. R., Souza L. S., Amaral L. A., Nogueira C. E. W. N. \& Roll V. F. B. 2015. Medidas comparativas do padrão morfométrico e perfil energético de éguas Crioulas no terço final da gestação, com diferentes escores corporais. Arquivos Brasileiros de Medicina Veterinária e Zootecnia. 67(3): 707-715.

McIlwraith, C. W. 2004. Developmental orthopedic disease: problems of limbs in young horses. Journal of Equine Veterinary Science. $24(11)$ : 475-479.

Pagan J. D., Geor R. J., Caddel S. E., Pryor P. B. \& Hoeskstra K. E. 2001. The relationship between glycemic response and the incidence of OCD in Thoroughbred weanlings: A field study. In: American Association of Equine Practitioners. 47:322-325.

Paz C. F. R., Paganela J. C., dos Santos C. A., Nogueira C. E. W. \& Faleiros R. R. 2013. Relação entre obesidade, insulina plasmática e posicionamento da falange distal em equinos da raça Crioula Arquivos Brasileiros de Medicina Veterinária e Zootecnia. 65(6): 1699-1705.

Peugnet P., Robles M., Wimel L., Tarrade A. \& Chavatte-Palmer P. 2016. Management of the pregnant mare and long-term consequences on the offspring. Theriogenology. 86(1): 99-109.

Pimentel A. M. H. 2017. O sexo no crescimento de potros da raça Crioula, do nascimento aos três anos de idade. Ciência Rural. 47(1).

Ralston S. L. 1996. Hyperglycemia/hyperinsulinemia after feeding a meal of grain to young horses With Osteochondritis Dissecans (OCD) Lesions. Pferdheilkunde Equine Medicine. 12: 320-322.

Robert C., Valette J-P. \& Denoix J. M. 2006. Correlation between routine radiographic findings and early racing career in French Trotters. Equine Veterinary Journal. 38(S36): 473-478.

Robles M., Nouveau E., Gautier C., Mendoza L., Dubois C., Dahirel M., Lagofun M-C. A., Lejeune J-P., Caudron I., Guenon I., Viguié C., Wimel L., BouraimaLelong H., Serteyn D., Couturier-Tarrade A. \& Chavatte-Palmer P. 2018. Maternal obesity increases insulin resistance, lowgrade inflammation and osteochondrosis lesions in foals and yearlings until 18 months of age. PLOS ONE. 13(1): 1-25.

Savage M.C. \& Jeffcott L. B. 1993. Effects of dietary energy and protein on induction of dyschondroplasia in foals. Equine Veterinary Journal. 16: 74-79.

Torres A. J., Nogueira C. E. W., Correa A., Brasil C. L., Finger I. S., Feijó J., Mousquer M. A., De Bastiani G., Pires Neves A. 2020. Foals developmental orthopedic disease associated with metabolic and biometric characterization of pregnant overweight Crioulo mares. Acta Scientiae Veterinariae. 48 : 1758. 